IMAGES IN CLINICAL PRACTICE

\title{
INTRACRANIAL CALCIFICATIONS WITH DANDY WALKER MALFORMATION
}

\author{
Sakthi Seethalakshmi P, Kumaravel KS, Sampathkumar P, Babu T, Rani SR \\ Department of Pediatrics, Government Mohan Kumaramangalam Medical College, Salem, Tamilnadu, India
}

ARTICLE HISTORY

Received 25 August 2018

Accepted 15 September 2018
A male child born of non-consanguineous marriage was admitted with severe microcephaly and seizures. There were no ocular, hematological, abdominal or cardiovascular abnormalities. CT brain showed cerebellar vermian agenesis, Dandy-Walker Malformation (DWM), lissencephaly, agenesis of corpus callosum, intracerebral-periventricular and basal ganglia calcification (Figure 1,2). TORCH infection was suspected but the serology for all TORCH components, repeated twice was negative. Maternal TORCH serology was also negative.

Figure 1. CT brain showing intracerebral and periventricular calcifications.

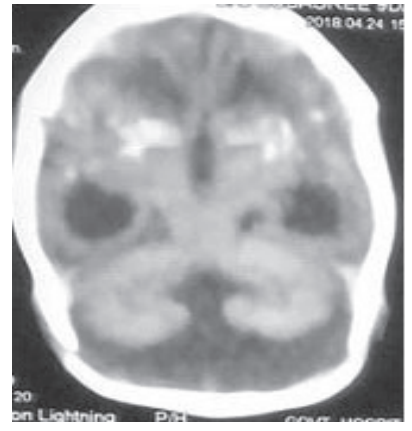

Figure 2. CT brain showing posterior fossa cyst communicating with fourth ventricle, cerebellar hypoplasia, ventriculomegly, lissencephaly and cortical atrophy

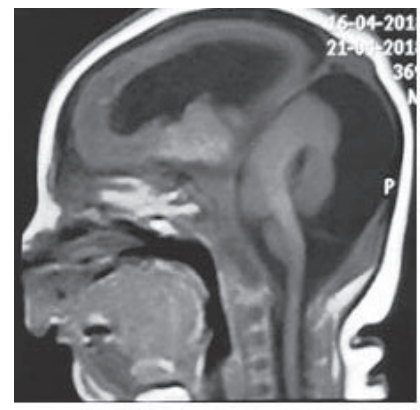

CONTACT Kumaravel KS

Email: Kumaravelks@Rediffmail.Com

Address for Correspondence: Kumaravel KS, 191A, Shankar Nagar, Salem 7,

Tamil Nadu - 636007.

(C)2019 Pediatric Oncall
What is the diagnosis?

\section{Discussion}

Pseudo TORCH syndrome (PTS) is a poorly defined heterogeneous group of disorder that is reported rarely in literature. It is an autosomal recessive condition which is clinically similar to congenital TORCH infections. It was first described by Baraitser et al. ${ }^{1}$ It is also known as Microcephaly Intracranial Calcification Syndrome and Baraitser - Reardon syndrome. ${ }^{1}$ The spectrum of clinical findings includes microcephaly, seizures, cerebral atrophy, intracranial calcifications, hepatosplenomegaly, thrombocytopenia, and developmental delay. However, microcephaly, intracranial calcifications, and cerebral atrophy are uniformly present. ${ }^{2}$ Prevalence is less than $1 / 1000000$. The clinical course is similar to that seen in patients after intrauterine infection. The intracranial calcifications in neonates can be seen in rare syndromes like AicardiGoutieres syndrome, Cockayne syndrome and Fetal akinesia deformation sequence. In all these syndromes the calcifications are diffuse and not confined to the periventricular region like that of Cytomegalovirus (CMV) infections. On the other hand, Dandy-Walker malformation presents with a large posterior fossa cyst, cerebellar vermis hypoplasia, and hydrocephalus. The malformation is often associated with agenesis of the corpus callosum, dysplasia of the brainstem, and cerebellar dysgenesis. But the presentation of DandyWalker Malformation with microcephaly and intracranial calcification is very rare.

The association between DWM and PTS has been reported by Cohen in 2012 and Patnaik in 2017.3,4 In our case, there were clinico-radiological features of CMV infection and radiological features of DWM. Though it may be necessary to confidently exclude the CMV infection by doing a PCR (Polymerase Chain Reaction), testing in cerebrospinal fluid for CMV antigens, the association of DWM is strongly against the diagnosis of CMV infection as they have not been reported with them. The management of these cases includes supportive care and there is no need for any shunt procedure as they are not associated with increased intracranial tension.

After four months of follow up, the child is seizure free on anti-epileptic drugs and has delayed developmental milestones.

\section{Compliance with Ethical Standards \\ Funding: None \\ Conflict of Interest: None}


References :

1. Baraitser M, Brett EM, Piesowicz AT. Microcephaly and intracranial calcification in two brothers. J Med Genet $1983 ; 20: 210-212$

2. Vivarelli R, Grosso S, Cioni M, Galluzzi P, Monti L, Morgese G, Balestri P. Pseudo-TORCH syndrome or BaraitserReardon syndrome: diagnostic criteria. Brain Dev. $2001 ; 23: 18-23$

3. Cohen MC, Karaman I, Squier W, Farrel T, Whitby EH. Recurrent pseudo-TORCH appearances of the brain presenting as "Dandy-Walker" malformation. Pediatr Dev Pathol. 2012;15:45-9

4. Patnaik A, Mishra SS, Das S. Extensive intracranial calcification of pseudo-TORCH syndrome with features of Dandy-Walker malformation. Asian J Neurosurg. 2017;12:541-3. 